\title{
Durotaxis behavior of bEnd.3 cells on soft substrate with patterned platinum nanoparticle array
}

\author{
Xiaomin $\mathrm{Wu}^{1,2}, \mathrm{Li} \mathrm{Li}^{1,2, *}$, Zecheng Lei ${ }^{1,2}$, Fan Yang ${ }^{1,2}$, Ri Liu ${ }^{1,2}$, Lu Wang ${ }^{1,2}$, Xinyao $\mathrm{Zhu}^{3} \&$ \\ Zuobin Wang ${ }^{1,2,4, *}$
}

\author{
${ }^{1}$ International Research Centre for Nano Handling and Manufacturing of China, \\ Changchun University of Science and Technology, Changchun 130022, China \\ ${ }^{2}$ Ministry of Education Key Laboratory for Cross-Scale Micro and Nano Manufacturing, \\ Changchun University of Science and Technology, Changchun 130022, China
}

${ }^{3}$ Key Laboratory of Mechanism Theory and Equipment Design of Ministry of Education, Tianjin University, Tianjin 300350, China

${ }^{4}$ JR3CN \& IRAC, University of Bedfordshire, Luton LU1 3JU, UK

*Corresponding author. (e-mail: wangz@cust.edu.cn; lil@cust.edu.cn)

\begin{abstract}
The directional arrangement of cells has crucial effect in tissue engineering fields such as wound healing and scar repair. Studies have shown that continuous nanostructures have directional regulatory effect on cells, but whether discontinuous nanostructures have the same regulatory effect on cells is also worthy of further study. Here, a series of discontinuous platinum nanoparticles (PtNPs) patterned on the surface of PDMS (PtNPs-PDMS\&Glass) and glass (PtNPs-Glass) substrates were developed to investigate the effect on bEnd.3 cell durotaxis. The laser interference lithography and nanotransfer printing method were employed to fabricate the substrates. It was found that about $80 \%$ cells orderly arranged on the PtNPs-PDMS\&Glass substrate, but only $20 \%$ cells orderly arrangement on the PtNPs-Glass substrate, and the number of cells on the PtNPsPDMS\&Glass substrate was 5 times more than that on the PDMS coated glass substrate (PDMS\&Glass). The results suggested that patterning PtNPs on the PDMS substrate not only provided the topographical guidance for cells just like continuous nanostructures but also promoted cell adhesion and growth. In addition, an improved whole cell coupling model was used to investigate and explain the cell durotaxis from the perspective of mechanism. These findings show the possibility of discontinuous nanostructures in regulating cell arrangement, and offer a useful method for the design of biological functional substrate, as well as help to understand the mechanism of cell durotaxis.
\end{abstract}

Keywords: PtNPs, cell durotaxis, nanotransfer printing methods, cell coupling model

\section{Introduction}

The extracellular matrix (ECM) exhibits complex network features ranging from nano- to microscale, such as various types of polysaccharides and proteins. These micro/nano features, including continuous (Abrams et al. 2002) and discontinuous structures (Inoue 1989), play an important role in the physiological activities of cells. The response of cells to micro/nano structures is in effect the response of cells to mechanical stimulation. The mechanical stiffness of the ECM influences the cellular functions including migration (Cortese et al. 2009), growth (Lien et al. 2009), differentiation 
(Zhang et al. 2020b), and even survival (Chen et al. 1997), as well as disease development such as liver fibrosis(Wells 2008), Alzheimer (Morawski et al. 2012), tumor cell metastasis (McDaniel et al. 2006) and arteriosclerosis (Orr et al. 2005). Hence, the effect of ECM stiffness on cells behavior have received extensive attention in recent years. However, the mechanism remains to be established, especially when stiffness patterning in nano-scale.

The complex stiff substrate with multiple stiffness gradients (Zhang et al. 2020a) and stable physicochemical properties (Shang et al. 2020) had been designed to act as ECMs for cell research in vitro. However, the complex structures are usually obtained by pouring compliant materials onto the surface of patterned structures and they are based on the fact that the underlying patterned layers can be sensed by the cells through superficial soft layer (Kuo et al. 2012), in which the patterned layers are mainly the topological structures prepared by chemical etching (Olszowska et al. 2017) or laser interference technique (Yu et al. 2010), while the soft layer is made of gel, such as the polyacrylic acid, or polyacrylamide. At the same time, the corresponding coupling models, such as the physical model of viscoelastic substrate (Chaudhuri et al. 2015) and whole cell migration simulator (Bangasser et al. 2017), for understanding the mechanism of cells in the sense-andresponse to the stiff substrate were also developed. However, due to the limitation of the compliant layer thickness and the characteristic size of the nanostructure, when the underlying patterned substrate nanostructure is discontinuous, the carefully designed substrate with abundant physiomechanical cues is likely to fail to regulate cell behavior. Moreover, when the substrate surface is patterned with discontinuous nanostructures, it is difficult for existing models to provide comprehensive theoretical support for the interaction between the cells and the substrate.

This paper focused on studying the effects of discontinuous nanostructures on cell behaviors. In this work, a method for preparing complex stiffness substrates with discontinuous PtNPs arrays on the surface of soft and stiff substrates was proposed. The substrates allow cells to directly contact with the stiff structures to explore the cell's response to the surrounding environment, as shown in Scheme 1. In the method, the PtNPs prepared by laser interference lithography were then transferred to the surface of the viscoelastic PDMS and glass substrates using the PMMA-mediated nanotransfer printing method. Furthermore, an improved whole cell coupling model was established to explain the cell durotaxis cultured on the substrate. The scanning electron microscopy (SEM) and fluorescence microscopy (FM) were employed to observe the growth behavior of cells.

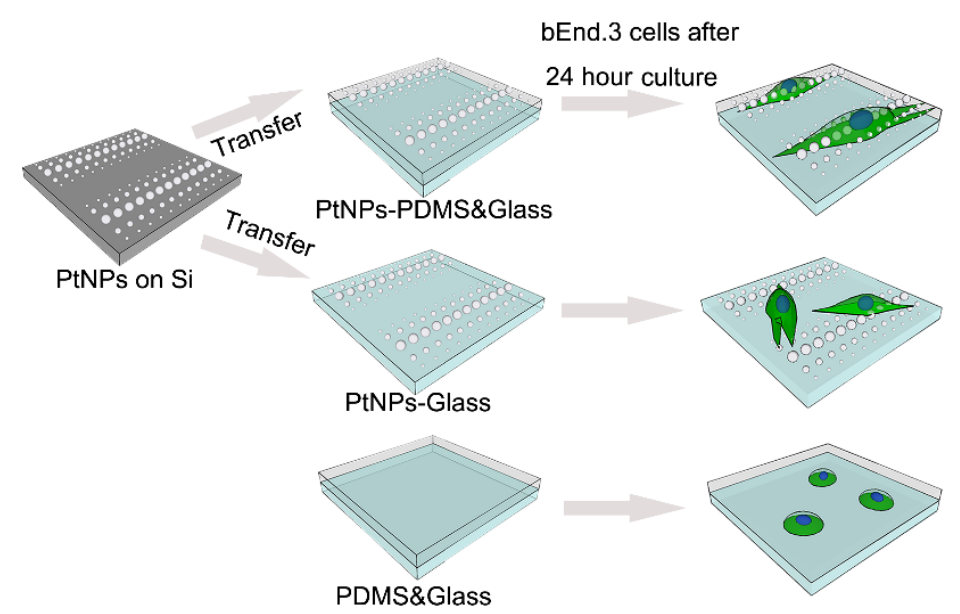

Scheme 1. Schematic of cell durotaxis behaviors on three substrates. 


\section{Methods}

Substrate preparation. Polydimethylsiloxane (PDMS) was composed of a pre-polymer (elastomer base) and a cross-linker (curing agent) (Cortese et al. 2009), which was purchased from Dow Corning (SYLGARD ${ }^{\mathrm{TM}}$ 184). The elastomer base and curing agent were mixed with the ratio of $50: 1(\mathrm{w} / \mathrm{w})$, and settled in the vacuum until all the trapped air bubbles were removed. Then, the mixture was poured on a previously cleaned glass substrate, and spun with the speed of $1000 \mathrm{rpm}$ for 60 seconds, followed by heating for about $1 \mathrm{~h}$ on the hot plate at $100^{\circ} \mathrm{C}$. The above steps of rotating and heating were repeated six times, and the last heating time was $1 \mathrm{~h}$. After the steps, the viscoelastic PDMS substrate was obtained.

The silicon wafers $\left(1.5 \times 1.5 \mathrm{~cm}^{2}\right)$ were ultrasonically cleaned orderly with acetone, ethanol and deionized water. The cleaned silicon wafers were treated with oxygen plasma, followed by platinum deposition with the thickness of $40 \mathrm{~nm}$. Here, the oxygen plasma treatment was used to increase the adhesion between the substrate and the coating layer. Pt particle arrays were prepared using the laser interference system, which consisted of the Nd: YAG laser (INNOLAS-SPITLIGHT 2000-10, 1064 $\mathrm{nm}, 8 \mathrm{~ns}$ pulse, Gaussian beam), two-beam interference system and Pt-plated silicon wafers. Among them, the laser beam diameter was $6 \mathrm{~mm}$, the laser fluence was $212 \mathrm{~mJ} / \mathrm{cm}^{2}$ and the interference period was $7 \mu \mathrm{m}$. A detailed description of the preparation of nanoparticle arrays is available in the literature (Li et al. 2015; Zhou et al. 2015).

In order to transfer the PtNPs to the target substrates (PDMS substrate and glass substrate), the fourstep transfer method is critical (Jiao et al. 2008). 4 wt \% PMMA (182265, ALDRICH) was spincoated on the source substrate (silicon wafers with Pt particle arrays) to load PtNPs (Li et al. 2015). The PMMA film was peeled-off from the source substrate, then it was attached to the target substrate, and the PtNPs were released to the target substrate at last. To release the PtNPs onto the target substrate, PMMA was removed with the oxygen plasma treatment for about $2 \mathrm{~h}$. Here, although there was no transfer of PtNPs on the surface of PDMS substrate in the control group, the PDMS\&Glass substrate with transferred PMMA film was also treated with plasma for $2 \mathrm{~h}$ like the other two substrates to minimize the unnecessary difference among the three substrates.

Cell culture. bEnd.3 cells, harvested from mouse brain microvascular endothelial cells, were obtained from American Type Culture Collection (ATCC). As an important part of blood-brain barrier, bEnd. 3 cells play an important role in preventing foreign bodies from entering and ensuring the stability of brain microenvironment (Watanabe et al. 2013). $50 \mu \mathrm{L}$ of cell suspension $\left(60 \times 10^{4}\right.$ cells $\mathrm{mL}^{-1}$ ) was diluted to $3 \mathrm{~mL}$ of fresh culture medium and added to a petri dish with the PtNPsPDMS\&Glass substrate, PtNPs-Glass substrate and PDMS\&Glass substrate placed in advance. Then, they were maintained in the Dulbecco's modified Eagle medium (DMEM) (gibco by Thermo Fisher Scientific) containing D-Glucose, L-glutamine and sodium pyruvate, supplemented with $1 \%$ Penicillin-streptomycin (Solarbio) and $10 \%$ fetal bovine serum (FBS) at $37^{\circ} \mathrm{C}$ in $5 \% \mathrm{CO}_{2}$ humidified atmosphere. Before the cells were seeded, the three substrates were rinsed with phosphate buffered saline (PBS, 0.01M) (Solarbio) three times, each time for $5 \mathrm{~min}$.

Immunofluorescence. After 2-day incubation, samples washed with PBS were then fixed in 4\% 
formaldehyde solution (Solarbio) at room temperature for about $10 \mathrm{~min}$. After washed two or three times with PBS, the samples were treated with 5\% Triton X-100 for 5 min to increase cell membrane permeability, and then stained with FITC-Phalloidin (Solarbio) for $30 \mathrm{~min}$. Subsequently, samples were incubated with 4',6-diamidino-2-phenylindole (DAPI) (Solarbio) for $30 \mathrm{~s}$. In the experiment, the samples were rinsed two or three times for $10 \mathrm{~min}$ by PBS in each staining step.

Atomic force microscope (AFM) characterization of substrates. AFM (JPK, Nano Wizard 3, Germany) measurements of Young's moduli of the three substrates were performed using the QI (quantitative imaging) mode. A type of triangular shaped MLCT probe (Bruker AFM probe, Germany) with the spring constant of $0.07 \mathrm{~N} / \mathrm{m}$ was used for the PtNPs-PDMS\&Glass substrate and PDMS\&Glass substrate test with the pixel time of $50 \mathrm{~ms}$, while a type of rectangular shaped OTESPA probe (Bruker AFM probe, Germany) with the spring constant range of 12-103 N/m was utilized for the PtNPs-Glass substrate test with the pixel time of $22 \mathrm{~ms}$. All the collected data were processed by JPK Data Processing Software (JPK, Nano Wizard 3, Germany).

SEM characterization of cells. To characterize the growth behavior of bEnd. 3 cells cultured on the PtNPs-PDMS\&Glass substrate, PtNPs-Glass substrate and PDMS\&Glass substrate using SEM (FEI: Quanta $250 \mathrm{FEG}$ ), a method of cell fixing for SEM imaging was necessary. First, an overnight fixing with $4 \%$ glutaraldehyde in the aqueous solution at $4^{\circ} \mathrm{C}$ was performed, followed by two or three times washed with PBS. Then, the dehydration in $50 \%, 70 \%, 80 \%, 90 \%, 95 \%$ and $100 \%$ concentrations of ethanol was done orderly, and the cells were then dehydrated further in the tertbutanol at $-20^{\circ} \mathrm{C}$ overnight and dried in a vacuum oven. After the dehydration, the samples were coated with a layer of gold $(5 \mathrm{~nm})$.

Quantification of cells. Fluorescence imaging was performed using an inverted microscope with the objective magnification of $20 \times$ (DS-Ri2, Nikon, Japan), and the cell deviation angle and cell number quantification were analyzed by Image $J$ software. The angle between the long axis direction of the cell and the horizontal line (the direction of PtNPs was horizontal) was defined as the cell deviation angle. Two hundred cells $\left(0.58 \times 0.58 \mathrm{~mm}^{2}\right.$ area $)$ were selected from each of the three substrates to calculate the deviation angle. When the angle between the cells and the horizontal line was greater than $90^{\circ}$, the supplementary angle was selected as its deviation angle. The total number of cells in the $0.58 \times 0.58 \mathrm{~mm}^{2}$ region on the 5 samples was counted and then averaged to obtain the mean of the cells on the three substrates using the Image $\mathbf{J}(\mathrm{NIH})$ software.

\section{Results and Discussion}

Preparation and characterization of substrates. The PtNPs-PDMS\&Glass and PtNPs-Glass substrates were prepared by Poly(methyl methacrylate) (PMMA)-mediated nanotransfer printing method (Jiao et al. 2008). A high-power pulsed Nd: YAG laser with the wavelength of $1064 \mathrm{~nm}$ was used for the preparation of PtNPs (Li et al. 2015; Zhou et al. 2015). Fig. 1 shows the steps of the fabrication process of PtNPs-PDMS\&Glass and PtNPs-Glass substrates. First, the PMMA solution ( $4 \mathrm{wt} \%$ ) was spin coated on the top surface of silicon wafer patterned with PtNPs (source substrate) at the speed of $2000 \mathrm{rpm}$, followed by treating in an oven at $25^{\circ} \mathrm{C}$ for $1 \mathrm{~h}$, and then baking on a hot plate at $115^{\circ} \mathrm{C}$ for $15 \mathrm{~min}$ to load the PtNPs onto the PMMA mediator (Feng et al. 2011). At the 
same time, the PDMS solution was poured on the previously cleaned glass substrate $\left(2 \times 2.6 \mathrm{~cm}^{2}\right)$ and rotated at $1000 \mathrm{rpm}$, and then baked at $100^{\circ} \mathrm{C}$ until the film was cured. The steps of rotating and baking were repeated six times (target substrate). The $\mathrm{KOH}$ aqueous solution (Feng et al. 2011) (1M, $80^{\circ} \mathrm{C}$ ) played an important role in the process of peeling off the PMMA-PtNPs film from the source substrate. To make the peel-off film firmly adhering to the target substrate, further baking at $50{ }^{\circ} \mathrm{C}$ was followed (Jiao et al. 2008). To release the PtNPs to the target substrate, the oxygen plasma cleaner (Diener electronic, Germany, $210 \mathrm{~W}, 2 \mathrm{~h}$ ) was used to remove the PMMA film at last.

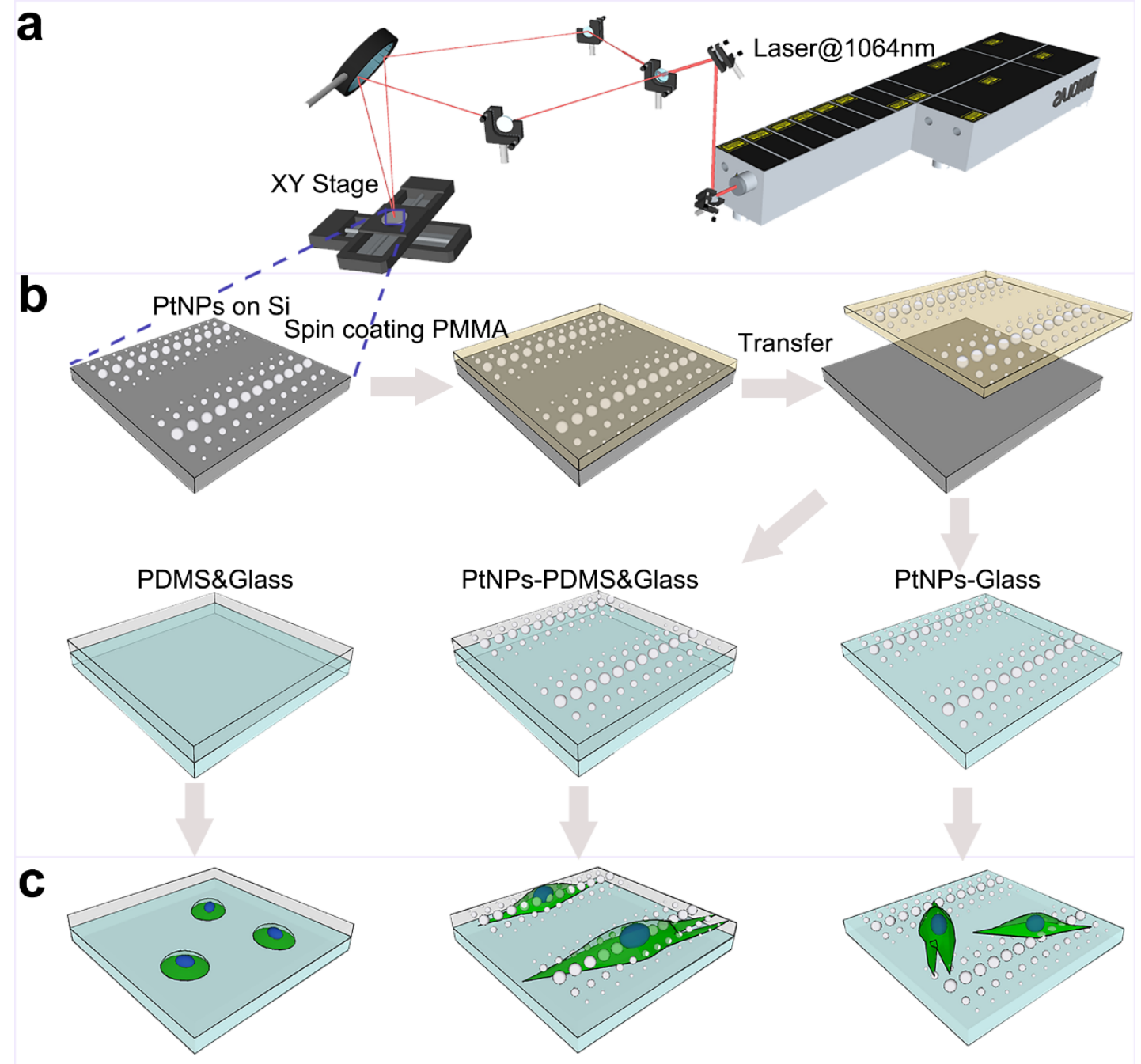

Figure 1. Schematic diagrams showing the fabrication processes of PtNPs-Glass, PtNPs-PDMS\&Glass and PDMS\&Glass substrates. (a) Schematic diagram of dual-beam laser interference setup for patterning PtNPs on a silicon substrate. (b) Flow diagram of the PMMA-mediated nanotransfer printing method. (c) Schematic diagram of cell growth on the three substrates. 
a
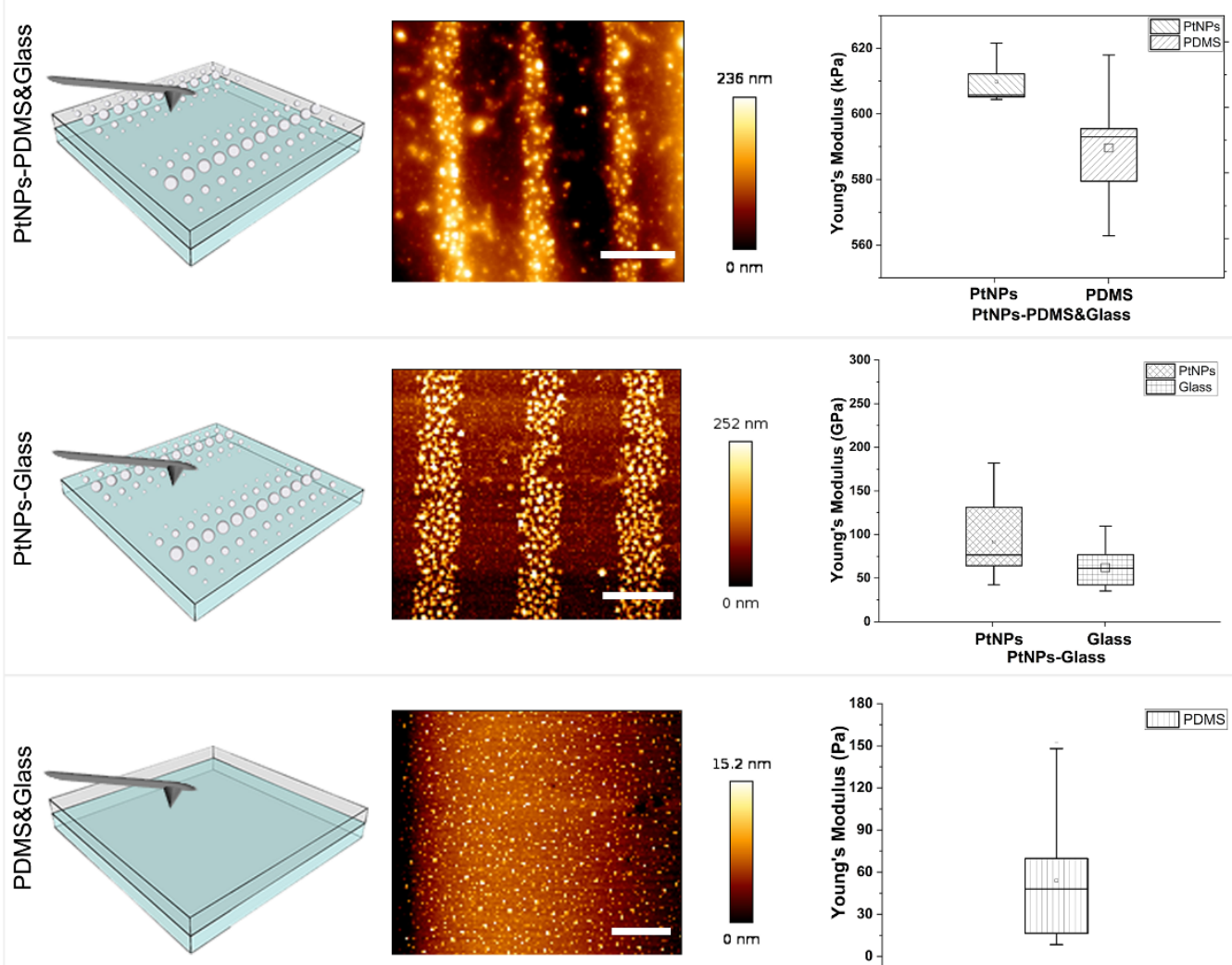

C
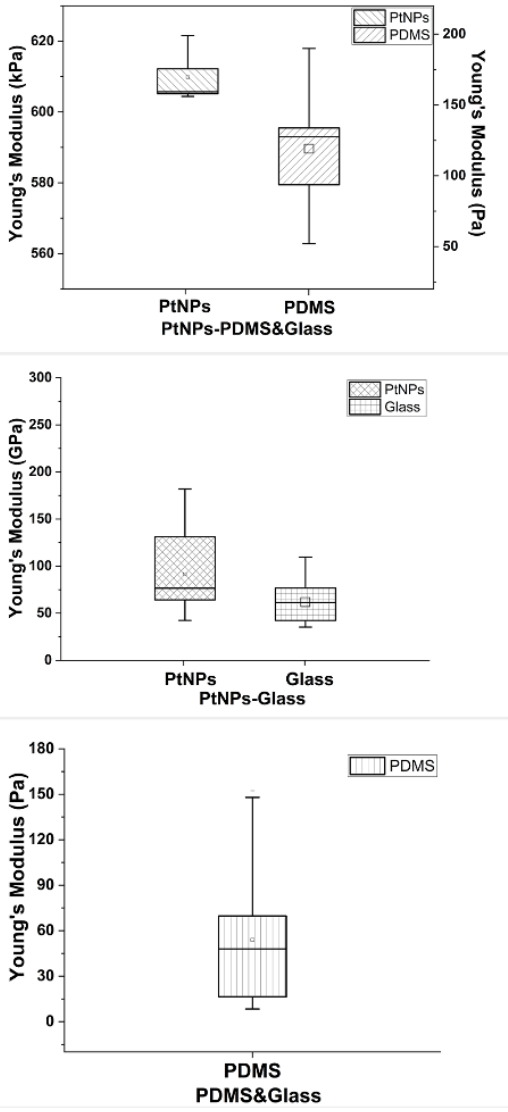

Figure 2. AFM characterization of three substrates. (a) Schematic diagrams of AFM characterization of three substrates. (b) The height maps of the three substrates; Scale bar: $5 \mu \mathrm{m}, 5 \mu \mathrm{m}$ and $2 \mu \mathrm{m}$, respectively. (c) Box-plots of Young's moduli for the three substrates.

The mechanical properties of the PtNPs-PDMS\&Glass substrate, PtNPs-Glass substrate and PDMS\&Glass substrate were tested by AFM. The precise AFM mapping allows the quantitative characterization of Young's moduli of the substrates. As shown in Fig. 2, the significant difference in the magnitude of Young's moduli is observed on the PtNPs-PDMS\&Glass substrate. On the substrate, the Young's modulus of PtNPs fluctuates between 605-620 kPa, with the mean value of $610 \mathrm{kPa}$, while the PDMS around the PtNPs array exhibits scattered data ranged from $50 \mathrm{~Pa}$ to 200 $\mathrm{Pa}$ with the mean value of $120 \mathrm{~Pa}$. However, on the PtNPs-Glass substrate, there is no significant difference in the magnitude of mean Young's moduli between PtNPs and glass substrate: $90 \mathrm{GPa}$ and $60 \mathrm{GPa}$, respectively. The Young's modulus of PDMS exhibits scattered data ranged from $10 \mathrm{~Pa}$ to $150 \mathrm{~Pa}$ with the mean value of $50 \mathrm{~Pa}$ when it is on the PDMS\&Glass substrate. The results show that the Young's modulus of PtNPs patterned on the PDMS and glass substrates are quite different (610 kPa and $90 \mathrm{GPa}$, respectively), indicating that the stiffness of the substrate underlying the PtNPs strongly affects the measurement of the Young's modulus of PtNPs (Abalymov et al. 2020). In addition, there are some differences in the magnitude of Young's modulus about PDMS between PtNPs-PDMS\&Glass and PDMS\&Glass substrates (120 Pa and $50 \mathrm{~Pa}$, respectively), which may be caused by the introduction of impurities in the process of transferring the PtNPs array. The height of the PtNPs was also estimated by AFM height map, ranged from $236 \mathrm{~nm}$ to $252 \mathrm{~nm}$, as shown in Fig. 2(b). The quantitative characterization of substrates shows that the substrates with complex 
stiffness can provide abundant physio-mechanical cues during cell culture, and the quantitative Young's moduli can provide data support for the understanding of cell durotaxis mechanism in this work.

Improved whole cell equivalent model. The effects of complex stiff substrates on cell durotaxis based on the molecular-clutch model (Chan and Odde 2008) were investigated. Due to the substrate prepared herein consisting of discontinuous PtNPs and PDMS substrate, the improved whole cell equivalent model of PtNPs-PDMS\&Glass substrate was proposed to be appropriately adapted, as shown in Figs. 3(a) and (b).

First, based on the previous F-action and substrate coupling model, the consideration of physical link between the cell body and the substrate was added, which was helpful for understanding the cell growth behavior at the cellular level (Bangasser et al. 2017). Then, the junction of the PtNPs on the PDMS substrate was simulated as three springs with different directions. Among them, the middle vertical spring provided the elastic force perpendicular to the PDMS substrate, while the left and right springs provided not only the vertical elastic force, but also the mechanical force of the interaction between adjacent PtNPs (Oria et al. 2017). Next, the PDMS substrate with viscoelasticity was modelled as an array of nodes connected by a Burgers structural unit model representing a viscoelastic substrate with stress relaxation (Chaudhuri et al. 2015).

In this adapted model, the F-actin bundles were connected to the substrate by a motor-clutch and driven by the myosin motor to produce the retrograde flow. The cellular traction force was transmitted to the substrate through the motor clutch, causing the deformation of soft substrate. The energy that cell-generated forces exerted on the substrate was expected to exhibit a decrease over time since the flow and remodeling of the stress-relaxed substrate, relaxing the resistance of cellular traction forces (Chaudhuri et al. 2015). This phenomenon caused the resistance of the cell traction to be insufficient to restore the deformation of the basement, so that the surface of the substrate around the cells was wrinkled (Fig. 3(b), white arrows). The appearance of wrinkles on the surface of the substrate in turn verified the assumption of the viscoelastic PDMS substrate. The proposed model provides theoretical support for explaining the durotaxis of cells on the complex viscoelastic substrate in this work. 
a

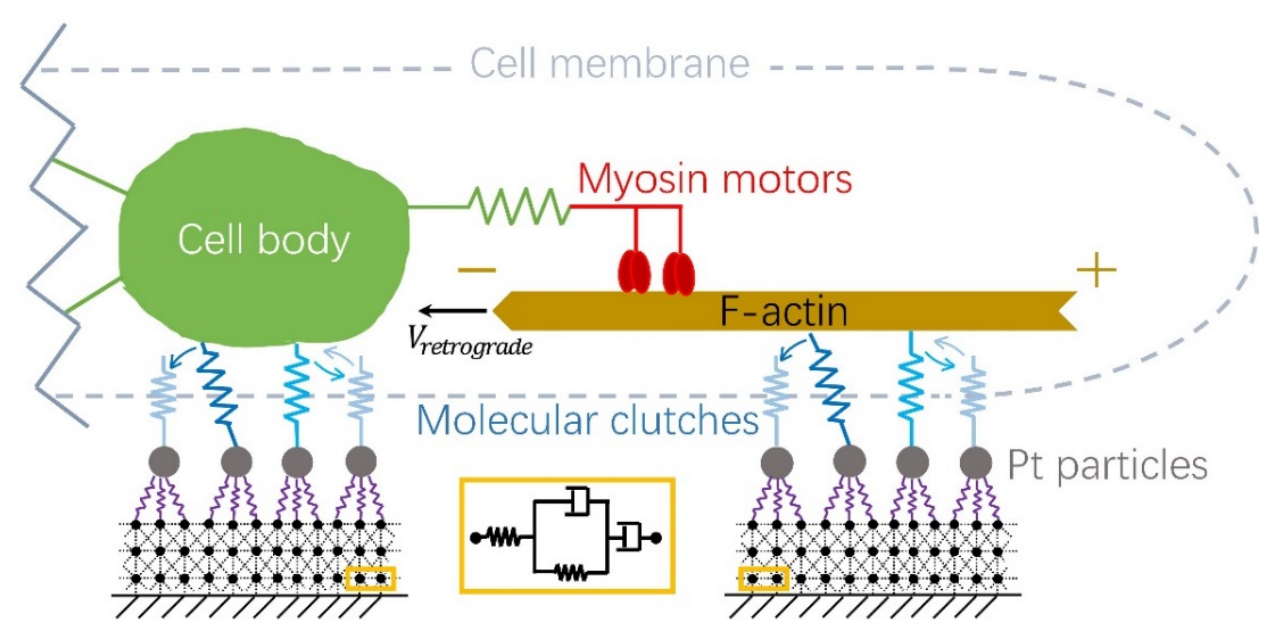

b

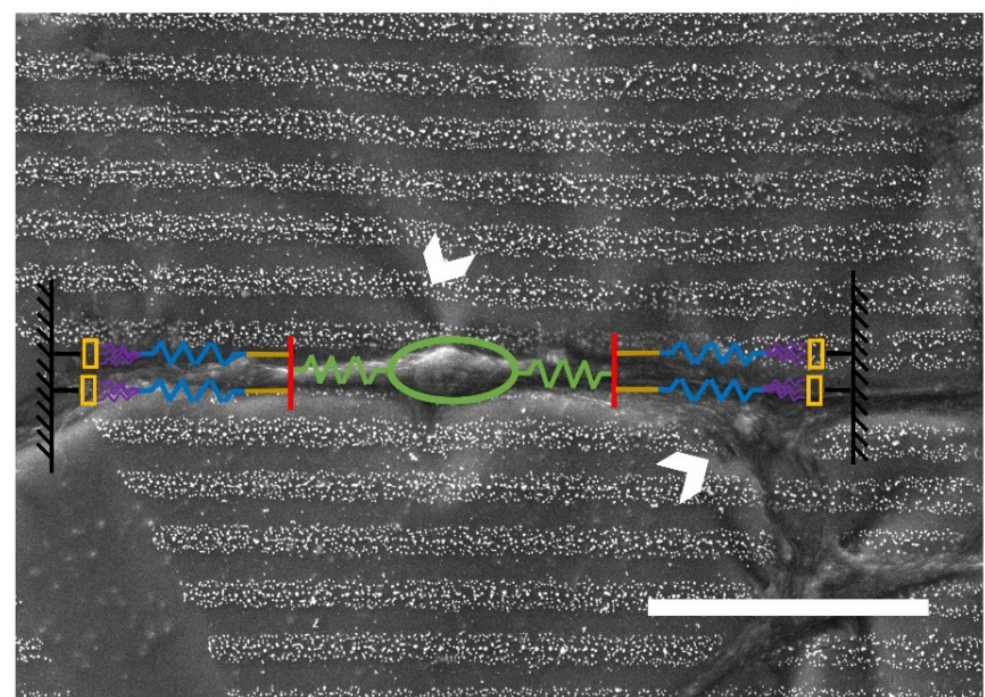

Figure 3. Improved whole-cell equivalent model of PtNPs-PDMS\&Glass substrate. (a) Schematic depiction of cell body and F-action attached to the viscoelastic PDMS substrate by molecular-clutch model. (b) Schematic of the improved whole-cell equivalent model overlaid on the top of an SEM image of bEnd.3 cells. The white arrows indicate the locations of some wrinkles on the surface of the PDMS substrate. Scale bar: $30 \mu \mathrm{m}$.

Growth behavior and mechanism of cells on substrates. After the release of the nanomaterial to the target substrates, the surfaces were rinsed by PBS solution before the culturing of cells. Both PtNPs-Glass and PDMS\&Glass acted as the control groups of PtNPs-PDMS\&Glass. Fig. 4 shows the growth behavior of cells on the substrates. The equivalent models of three types of substrates are shown in Fig. 4(a), in which the PtNPs-Glass substrate can be simulated as thin springs, representing the connection between PtNPs, and thick springs, representing the glass substrates in series (Oria et al. 2017). 
a

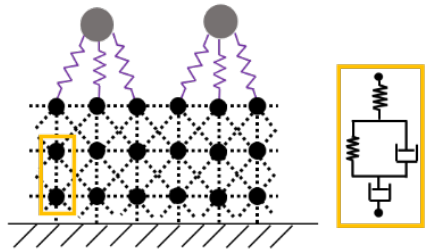

PtNPs-PDMS\&Glass

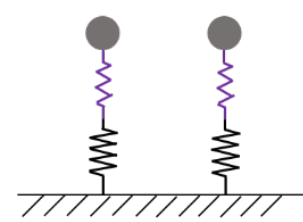

PtNPs-Glass

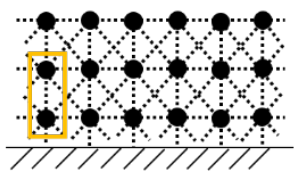

PDMS\&Glass

b

C
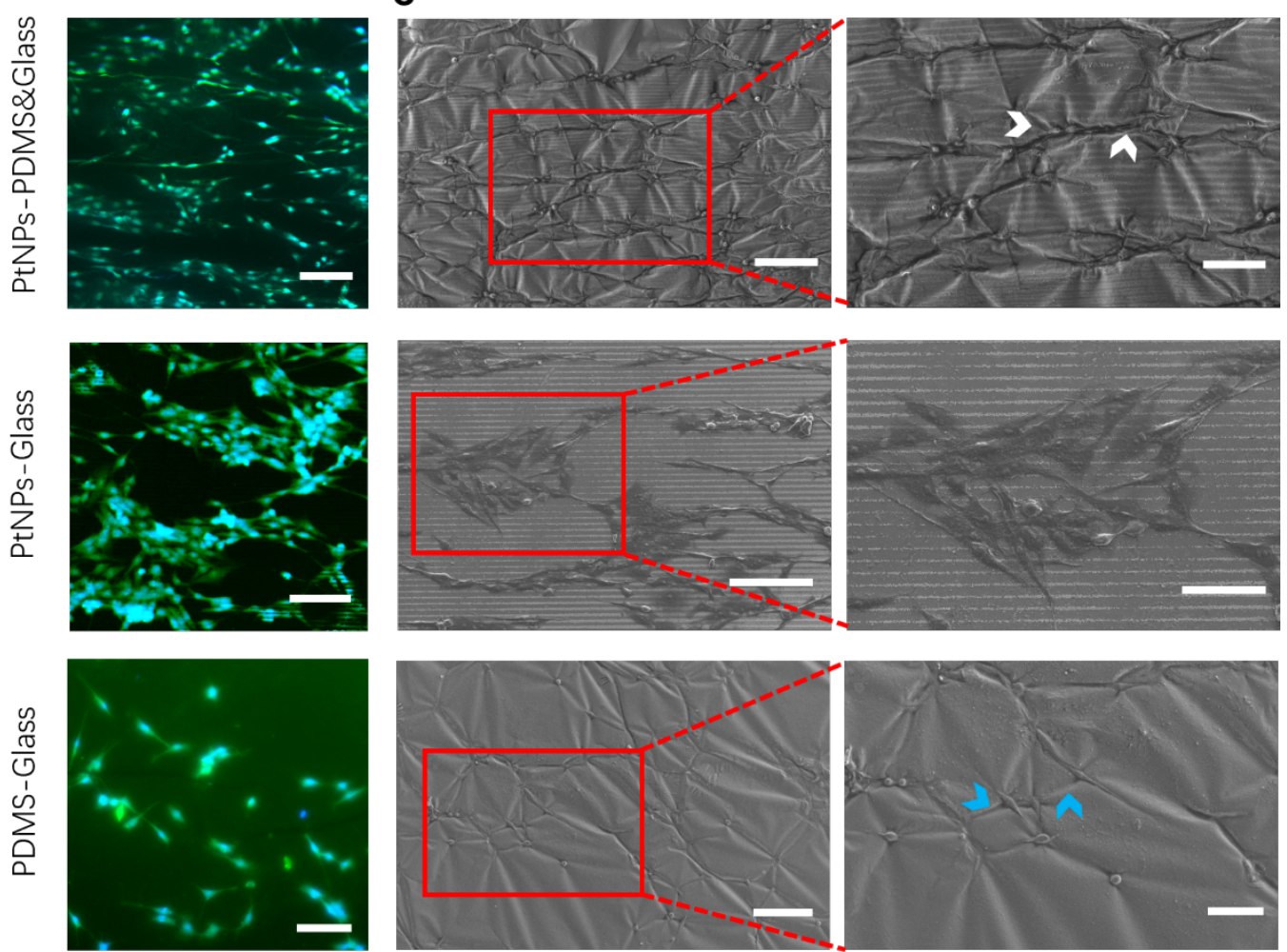

d

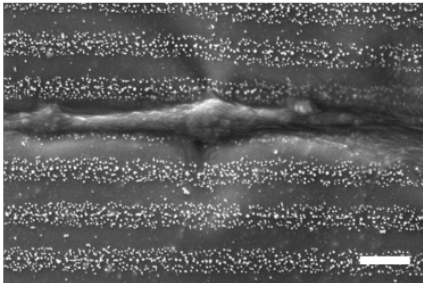

PtNPs-PDMS\&Glass

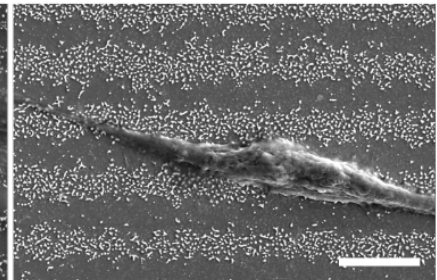

PtNPs-Glass

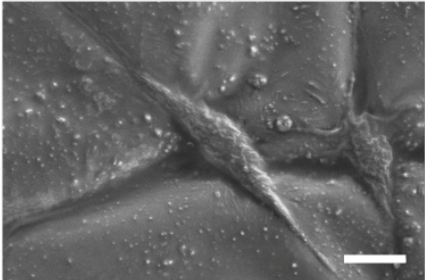

PDMS-Glass

Figure 4. Growth behaviors and morphological characteristics of bEnd.3 cells on different substrates. (a) The equivalent models of the three substrates. Notes: The yellow box in the equivalent model of the PDMS\&Glass substrate on the right is the same as that of the PtNPs-PDMS\&Glass substrate on the left. (b) Fluorescence microscope images of cell growth behaviors on the three substrates. Scale bar: $100 \mu \mathrm{m}$. (c) Left, SEM images of cell growth behavior on the three substrates. Scale bar: $100 \mu \mathrm{m}$; Right, the magnified SEM images of red boxed areas on the left. Scale bar: $50 \mu \mathrm{m}$. (d) The magnified SEM images of single cell growth behaviors on the three substrates. Scale bar: $10 \mu \mathrm{m}$.

As shown in Figs. 4(b-d), the growth behaviors of bEnd.3 cell on the three types of substrates were exhibited. Among them, the SEM images show the morphological changes of cells in response to the substrates, while the FM images show the orientations of the cytoskeletons. The cells aligned 
along the PtNPs array are slightly embedded in the PDMS on the PtNPs-PDMS\&Glass substrate. The cells cultured on the PtNPs-Glass substrate appear unregulated growth and good spread. The cells show unregulated growth and they are also slightly embedded in the PDMS on the PDMS\&Glass substrate (Yeung et al. 2005), and the number of cells is significantly less than those on the other two substrates. In addition, the wrinkles (Fig. 4(c), white arrows and blue arrows) appeared on the PtNPs-PDMS\&Glass and PDMS\&Glass substrates are due to the effect of stressrelaxing that the substrate cannot rebound to the original position when the cell traction applied to the substrate is released. It confirms the assumption of viscoelastic substrate in the coupling model from the perspective of experiment.

It is known that cells sense the stiffness of the ECM by integrating proteins and their complexes on the cell membranes, and then adjust their own behaviors in response to the stiffness cues ("durotaxis") (Lo et al. 2000). To further understand the mechanism of cell behavior, the experimental results on the three substrates were analyzed. By comparing the cells on the PtNPsPDMS\&Glass and PtNPs-Glass substrates, it is concluded that only the PtNPs patterned on the surface of PDMS substrate (soft) can produce the topographic guidance similar to the continuous structure to the cells. This may be due to the strong contrast of the Young's moduli between the PDMS substrate and PtNPs (120 Pa and $610 \mathrm{kPa}$, respectively), and the durotaxis of the cells promotes them growing along the PtNPs arrays. However, the Young's moduli of PtNPs and glass substrate on the PtNPs-Glass substrate have the same magnitude (90 GPa and $60 \mathrm{GPa}$, respectively) and little difference in the mean values. Therefore, the cells cultured on the PtNPs-Glass substrate are not controlled by the PtNPs arrays. Compared with the PDMS\&Glass substrate, the cells cultured on the PtNPs-PDMS\&Glass substrate were aligned along the PtNPs array and the number of cells was significantly increased, which suggested that patterning PtNPs arrays on the PDMS substrate regulated the cell growth behavior and promoted the cell adhesion. Based on the above analysis, the mechanism of cell durotaxis on the PtNPs-PDMS\&Glass substrates was proposed. Firstly, the cell was randomly adhered to the substrate surface after seeding. Due to the strong contrast of the Young's moduli between the PDMS and PtNPs, the cell durotaxis feature enabled cells to grab PtNPs. At the same time, the PtNPs existed in the form of arrays, which eventually led to the orderly growth of cells in the same direction as the PtNP arrays. In this study, the cell's behavior of grasping particles in the soft matrix is consistent with the results of other studies (Abalymov et al. 2020). The difference is that the nanoparticles here are arranged in order, which can regulate the orderly growth of cells.

Quantification of cell deviation angle and cell number. Exploring the influence of discontinuous PtNP arrays on the behavior of bEnd.3 cells, especially the arrangement of cells, is of great significance for studying blood-brain barrier diseases (Abbott et al. 2010). From the results of SEM and FM images, it can be seen that the behaviors of cells on the three substrates are obviously different. In order to visualize the phenomenon more intuitively, the deviation angles between the cells and the directions of PtNPs were analyzed using Image $\mathrm{J}$ software. The angle of $0^{\circ}$ represents that the cells align with the PtNPs, whereas the angle of $90^{\circ}$ represents the cells are perpendicular to the PtNPs. As shown in Figs. 5(a-c), the deviation angles for cell extensions on the PtNPsPDMS\&Glass substrate are concentrated in the $0^{\circ}-15^{\circ}$ range and almost no distribution in the range of $30^{\circ}-90^{\circ}$, which indicates that the cells on the PtNPs-PDMS\&Glass substrate are affected by 
PtNPs array. However, on the PtNPs-Glass substrate and PDMS substrate the cell deviation angles are ranged from $0^{\circ}$ to $90^{\circ}$, suggesting a uniform distribution of deviation angles, which proves that the cells grow randomly on the two substrates. Fig. 5(d) shows a histogram of the directionality of cell quantification on the three substrates. Obviously, the proportion of cells on the PtNPsPDMS\&Glass substrate in the region with the deviation angles of less than $15^{\circ}$ (ratio of $80 \%$ ) is significantly greater than those of the other two angle ranges of only $20 \%$. On the PtNPs-Glass substrate and PDMS\&Glass substrate, the percentages of cells in the $0^{\circ}-15^{\circ}, 15^{\circ}-45^{\circ}$, and $45^{\circ}-90^{\circ}$ regions are different but not dramatically, indicating that the cells grow disorderly. The results show that only the PtNPs patterned on the surface of the PDMS substrate have a topographical guidance effect on the cells. To demonstrate that the patterned PtNPs array on the surface of viscoelastic PDMS is conducive to cell adhesion, the number of cells on the three substrates is statistically analyzed using Image $J$ software, and the results are shown in Fig. 6 . The average number of cells on the PtNPs-PDMS\&Glass substrate is 300 , which is not much different from the average number of cells on the PtNPs-Glass substrate that is 364 . In contrast, the average number of cells cultured on the PDMS\&Glass substrate was only 63, which is significantly less than those on the other two substrates. Obviously, compared with the PDMS substrate, the average number of cells is increased about 5 times when the PDMS surface modified with PtNPs (stiff structure) and is equivalent to the number of cells on the PtNPs-Glass substrate (stiff substrate), which suggests that increasing stiff structures on the PDMS substrate promotes the cell adhesion. The results here are consistent with those of other studies (Kuo et al. 2012), which use microstructures to regulate cell behavior.

a

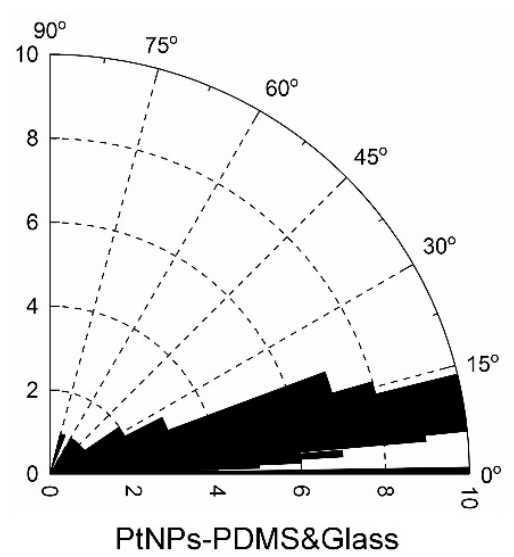

C

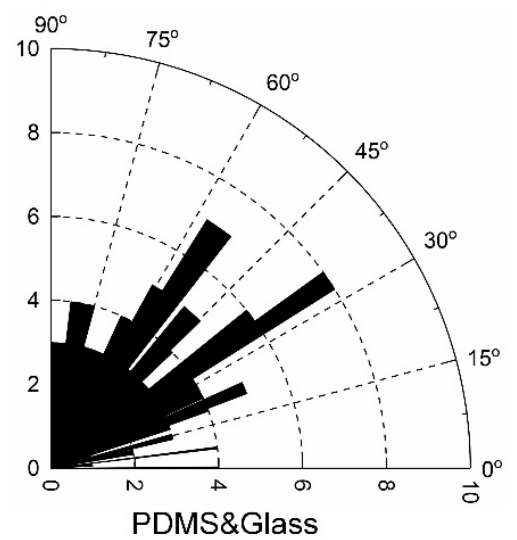

b
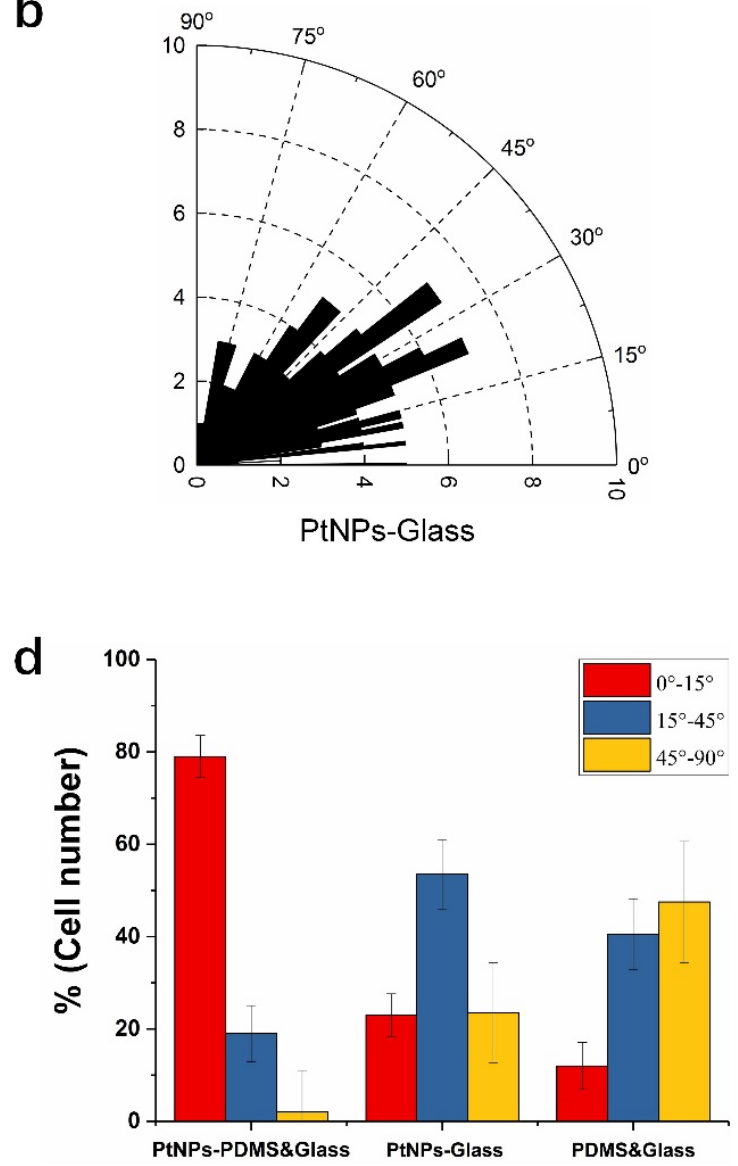

Figure 5. Cell angular distributions. (a-c) Visual representations of cell deviation angles on the three substrates 
( $n=200$ for each group). (d) Histogram of cell directionality. The error bars represent the standard deviations.

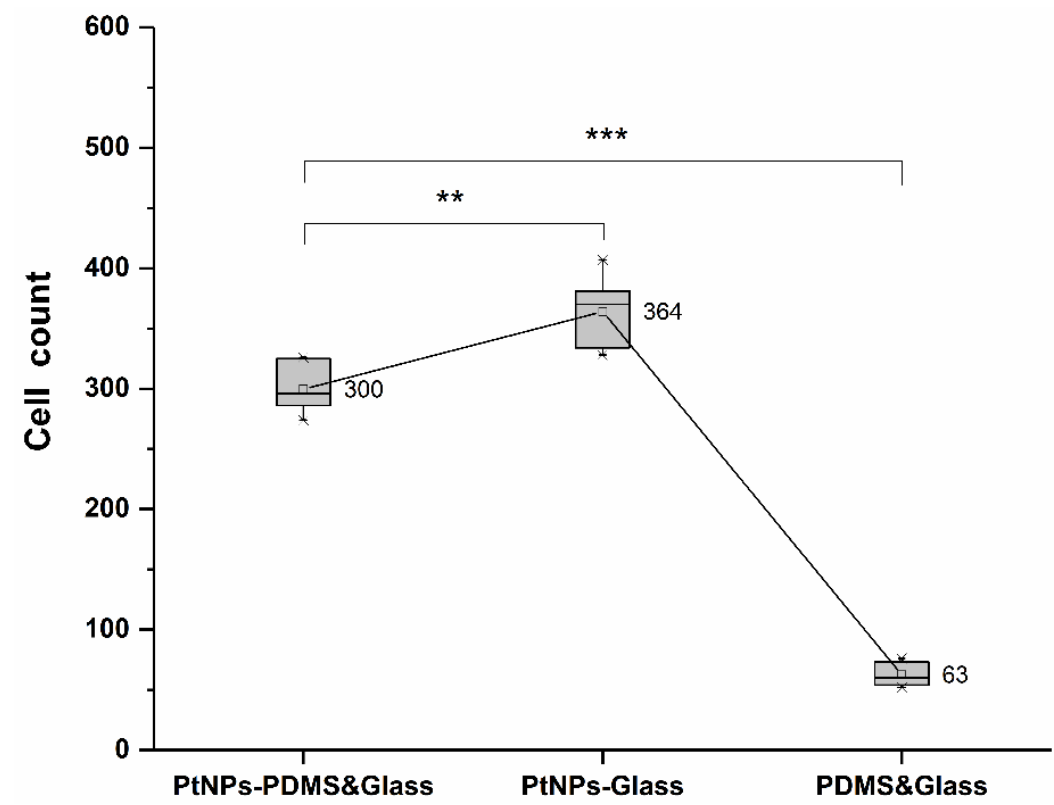

Figure 6. Cell counts. Results of cell counting on the three substrates. $* * * \mathrm{P}<0.001, * * \mathrm{P}<0.01$, t-test.

\section{Conclusions}

In summary, the research focused on the effect of discontinuous PtNPs arrays patterned on the substrates with different stiffness values on bEnd. 3 cell durotaxis. The cellular experimental results showed that the attachment of such stiff discontinuous PtNPs arrays on the surface of the PDMS substrate not only facilitated the cell adhesion and growth, but also topographically guided the cell growth directions just like the continuous structures. The results indicate that the strong contrast of Young's moduli between the PtNPs and the substrate is the premise for the discontinuous PtNPs arrays controlling the cell behavior. In addition, the improved whole cell coupling model provides a mechanism reference for explaining the results of cell experiments. Meanwhile, the wrinkles appear on the surface of PtNPs-PDMS\&Glass substrate, confirming the assumption of viscoelastic substrate in the coupling model. This work will advance the understanding of cell durotaxis mechanism, and the orderly arrangement of bEnd. 3 cells are helpful for studying the blood-brain barrier diseases. The flexible nanotransfer printing method offers a simple strategy for the probing of cell behaviors on soft materials, but the transfer mediators for different applications are still being investigated.

\section{Abbreviations}

PDMS: polydimethylsiloxane; PtNPs: platinum nanoparticles; PDMS\&Glass: PDMS coated glass; PtNPs-PDMS\&Glass: platinum nanoparticles patterned on the PDMS coated glass; PtNPs-Glass: platinum nanoparticles patterned on the surface of glass; DMEM: Dulbecco's modified Eagle medium; FBS: fetal bovine serum; PBS: phosphate buffered saline; DAPI: 4',6-diamidino-2phenylindole; PMMA: poly(methyl methacrylate); SEM: scanning electron microscopy; AFM: atomic force microscope; FM: fluorescence microscopy; ECM: extracellular matrix; bEnd.3: mouse brain microvascular endothelial cells.

\section{Acknowledgement}


This work was supported by National Key R\&D Program of China (No.2017YFE0112100), EU H2020 Program (MNR4SCELL No.734174), Jilin Provincial Science and Technology Program (Nos.20180414002GH, 20180414081GH, 20180520203JH, 20190702002GH, 20190201287JC and 20200901011SF), and "111" Project of China (D17017).

\section{Conflicts of Interest}

The authors declare that there is no conflict of interest.

\section{References}

Abalymov A, Meeren LVd, Saveleva M, Prikhozhdenko E, Dewettinck K, Parakhonskiy B, Skirtach AG (2020) Cells-grab-on particles: a novel approach to control cell focal adhesion on hybrid thermally annealed hydrogels ACS Biomaterials Science \& Engineering 6:3690-4323

Abbott NJ, Patabendige AAK, Dolman DEM, Yusof SR, Begley DJ (2010) Structure and function of the blood-brain barrier Neurobiology of Disease 37:13-25

Abrams GA, Bentley E, Nealey PF, Murphy CJ (2002) Electron microscopy of the canine corneal basement membranes Cells Tissues Organs 170:251-257

Bangasser BL et al. (2017) Shifting the optimal stiffness for cell migration Nature Communications 8:15313-15322

Chan CE, Odde DJ (2008) Traction dynamics of filopodia on compliant substrates Science 322:16871691

Chaudhuri O et al. (2015) Substrate stress relaxation regulates cell spreading Nature Communications 6:6364-6370

Chen CS, Mrksich M, Huang S, Whitesides GM, Ingber DE (1997) Geometric control of cell life and death Science 276:1425-1428

Cortese B, Gigli G, Riehle M (2009) Mechanical gradient cues for guided cell motility and control of cell behavior on uniform substrates Advanced Functional Materials 19:2961-2968

Feng D et al. (2011) Free-standing mesoporous carbon thin films with highly ordered pore architectures for nanodevices Journal of American Chemical Socity 133:15148-15156

Inoue S (1989) Ultrastructure of basement membranes International Review of Cytology-a Survey of Cell Biology 117:57-98

Jiao L, Fan B, Xian X, Wu Z, Zhang J, Liu Z (2008) Creation of nanostructures with poly (methyl methacrylate)-mediated nanotransfer printing Journal of American Chemical Socity 130:1261212613

Kuo CHR, Xian J, Brenton JD, Franze K, Sivaniah E (2012) Complex stiffness gradient substrates for studying mechanotactic cell migration Advanced materials 24:6059-6064

Li L et al. (2015) Fabrication of Pt nanowires with a diffraction-unlimited feature size by high-threshold lithography Applied Physics Letters 107:133104-133108

Lien SM, Ko LY, Huang TJ (2009) Effect of pore size on ECM secretion and cell growth in gelatin scaffold for articular cartilage tissue engineering Acta Biomaterialia 5:670-679

Lo CM, Wang HB, Dembo M, Wang Yl (2000) Cell movement is guided by the rigidity of the substrate Biophysical Journal 79:144-152

McDaniel SM, Rumer KK, Biroc SL, Metz RP, Singh M, Porter W, Schedin P (2006) Remodeling of the mammary microenvironment after lactation promotes breast tumor cell metastasis The American Journal of Pathology 168:608-620 
Morawski M, Brückner G, Jäger C, Seeger G, Matthews RT, Arendt T (2012) Involvement of perineuronal and perisynaptic extracellular matrix in Alzheimer's disease neuropathology Brain Pathology 22:547-561

Olszowska $\mathrm{K}$ et al. (2017) Three-dimensional nanostructured graphene: synthesis and energy, environmental and biomedical applications Synthetic Metals 234:53-85

Oria R et al. (2017) Force loading explains spatial sensing of ligands by cells Nature 552:219-224

Orr AW, Sanders JM, Bevard M, Coleman E, Sarembock IJ, Schwartz MA (2005) The subendothelial extracellular matrix modulates NF- $\mathrm{\kappa B}$ activation by flow: a potential role in atherosclerosis Journal of Cell Biology 169:191-202

Shang L et al. (2020) Nanotextured silk fibroin/hydroxyapatite biomimetic bilayer tough structure regulated osteogenic/chondrogenic differentiation of mesenchymal stem cells for osteochondral repair Cell Proliferation doi:10.1111/cpr.12917

Watanabe T et al. (2013) Paracellular barrier and tight junction protein expression in the immortalized brain endothelial cell lines bEND.3, bEND.5 and mouse brain endothelial cell 4 Biological \& Pharmaceutical Bulletin 36:492-495

Wells RG (2008) The role of matrix stiffness in regulating cell behavior Hepatology 47:1394-1400

Yeung $\mathrm{T}$ et al. (2005) Effects of substrate stiffness on cell morphology, cytoskeletal structure, and adhesion Cell Motility \& the Cytoskeleton 60:24-34

Yu F, Mücklich F, Li P, Shen H, Mathur S, Lehr C-M, Bakowsky U (2010) In vitro cell response to a polymer surface micropatterned by laser interference lithography Biomacromolecules 6:11601167

Zhang $\mathrm{R}$ et al. (2020a) Topographical regulation of stem cell differentiation by plantderived micro/nanostructures Nanoscale 12:18305-18312

Zhang S et al. (2020b) Neuron-like cell differentiation of hADSCs promoted by copper sulfide nanostructure mediated plasmonic effect driven by nearinfrared light Nanoscale 12:9833-9841

Zhou Z, Song Z, Li L, Zhang J, Wang Z (2015) Fabrication of periodic variable-sized Pt nanoparticles via laser interference patterning Applied Surface Science 330:65-70 